\title{
How to measure propagation velocity in cardiac tissue: a simulation study
}

\author{
Andre C. Linnenbank ${ }^{1,2 *}$, Jacques M. T. de Bakker ${ }^{1,2,3}$ and Ruben Coronel ${ }^{1}$ \\ ${ }^{1}$ Department of Experimental Cardiology, Heart Center, Academic Medical Center, Amsterdam, Netherlands \\ 2 ICIN, Utrecht, Netherlands \\ ${ }^{3}$ Department of Medical Physiology and Cardiology, UMCU, Utrecht, Netherlands
}

Edited by:

Gaetano Santulli, Columbia, USA

Reviewed by:

Godfrey Smith, University of

Glasgow, UK

Sandeep Pandit, University of

Michigan, USA

Michele Mario Ciulla, University of

Milan, Italy

*Correspondence:

Andre C. Linnenbank, Department of Experimental Cardiology,

Academic Medical Center,

Rm. K2-114, Meibergdreef 15, 1105

AZ Amsterdam, Netherlands

e-mail:a.c.linnenbank@amc.uva.nl
To estimate conduction velocities from activation times in myocardial tissue, the "average vector" method computes all the local activation directions and velocities from local activation times and estimates the fastest and slowest propagation speed from these local values. The "single vector" method uses areas of apparent uniform elliptical spread of activation and chooses a single vector for the estimated longitudinal velocity and one for the transversal. A simulation study was performed to estimate the influence of grid size, anisotropy, and vector angle bin size. The results indicate that the "average vector" method can best be used if the grid- or bin-size is large, although systematic errors occur. The "single vector" method performs better, but requires human intervention for the definition of fiber direction. The average vector method can be automated.

Keywords: activation mapping, conduction velocity, single vector method, average vector method, simulation, anisotropy

\section{INTRODUCTION}

The assessment of the conduction velocity of the cardiac impulse is an important aspect of the study of arrhythmogenesis, especially where it involves arrhythmias based on reentry (Kleber et al., 1986; Janse and Wit, 1989). Slow conduction facilitates reentrant arrhythmias, because it causes a reduction of the wavelength (Mines, 1914). Heterogeneity of conduction velocity and of excitability may provide a substrate for the occurrence of unidirectional block, which is essential for the onset of reentrant activation (Arita and Kiyosue, 1983; Coronel et al., 2009).

Spread of activation in myocardial tissue depends on the myocardial fiber direction (anisotropy). It is fastest in the direction of the myocardial fibers and slow transverse to fiber direction. The normal anisotropic ratio (longitudinal conduction velocity divided by transverse conduction velocity) is for ventricular myocardium about 2:1 (Spach et al., 1981). The difference is caused by the shape of the cardiomyocyte in combination with the preferential localization of connexins at the short sides of the myocytes. As a result of cardiac pathology, the dimensions of the myocyte may change (Wiegerinck et al., 2006), the localization of gap junctions may change toward the lateral sides of the myocytes (lateralization) (Peters et al., 1997) and the extracellular matrix (collagen content) may increase (Weber and Brilla, 1991). Myocardial scarring and fibrosis cause lateral isolation of the myocytes leading to an increase of local anisotropic ratio (Spach et al., 1981; Frazier et al., 1988; de Bakker et al., 1993; Kawara et al., 2001) and of the facilitation of current-to-load mismatch. The latter is important for the generation of unidirectional conduction block (Hoogendijk et al., 2010). In addition, various sodium channel mutations (Remme et al., 2006) and heart diseases (Valdivia et al., 2005) are associated with a decrease in peak sodium current and conduction velocity. An increase in anisotropy is also considered proarrhythmic (Wilders et al., 2000).

For the study of arrhythmia mechanisms the determination of the longitudinal and transverse conduction velocity $\left(\mathrm{CV}_{1}\right.$ and $\mathrm{CV}_{\mathrm{t}}$ ), therefore, is essential. Ideally, conduction velocities are calculated from high density surface mapping (either electrically or optically) studies following pacing from the center of the grid of mapping electrodes (Kleber et al., 1986). Under these conditions the activation wave shows an ellipsoid spread of activation starting from the central pacing site. At larger distances from the pacing site the surface activation pattern may show signs of epicardial (or endocardial) breakthrough following intramural propagation, resulting in unrealistically high calculated conduction velocities.

Two methods are commonly used to estimate conduction velocities (Figure 1). The "single vector" method is a more or less manual approach and consists of identifying recording sites on an inner and outer ellipsoid on a line perpendicular to the isochronal lines along the short and long axis of the ellipsoids. The recording sites are selected along a line perpendicular to the isochronal lines (Kleber et al., 1986). $\mathrm{CV}_{1}$ and $\mathrm{CV}_{\mathrm{t}}$ are then calculated from the difference in timing and the known distance between the recording points. Figure $2 \mathrm{C}$ shows the isochrones and the longitudinal end transversal vectors as they might be chosen in a manual procedure. Data recorded form a dog heart with a 13 by 16 electrode grid with $0.5 \mathrm{~mm}$ distance between the electrodes (Coronel et al., 2010) shown in Figure 2A.

The "average method" divides the whole grid in smaller subgrids (typical $3 \times 3$ or $5 \times 5$ recording points). Using the activation times in a subgrid, the best fitting vector (direction and 
velocity) of the activation front at the central point of the subgrid is estimated. This procedure yields a local activation vector for every point except for border points. (An example of vectors computed from the data in Figure 2A is shown in Figure 2B). These local activation vectors are collected in bins of vectors in the same direction. The average length of the vectors in a bin is used as the velocity in that direction. The average vectors with the largest and smallest conduction velocity value are selected as the longitudinal and transversal velocity in the grid.

Preliminary tests of the average vector method revealed that bins could be empty if the bin-size was too small. In particular in the direction of the fibers. Larger bin sizes on the other hand could result in bias if vectors were included that were not in pure transverse or longitudinal directions. The single vector method depends on selecting just 2 points, which might result in a larger SD. It is not clear which of the two methods performs better for the calculation of $\mathrm{CV}_{1}$ and $\mathrm{CV}_{\mathrm{t}}$. We therefor performed a simulation study to investigate the effect of bin size, grid size, anisotropy,
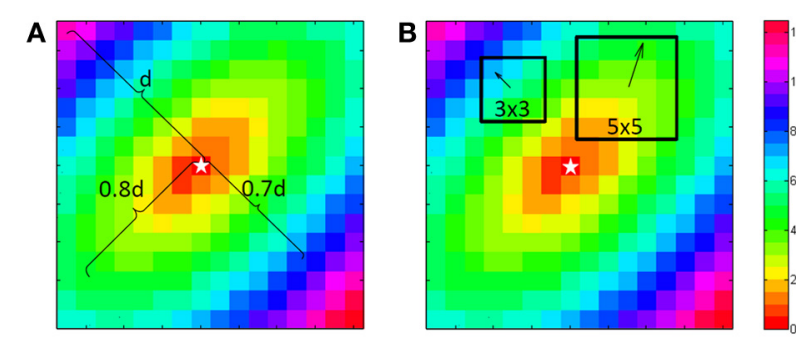

FIGURE 1 | Computation of the longitudinal and transversal velocities. For the single vector method a point is chosen at $80 \%$ of the length from the central "stimulus" (star shape) to the corner in the direction of the fibers. This is used to define the longitudinal velocity. At right angles at $70 \%$ another point is chosen for the transversal velocity. Because this is a simulation both can use the center as the starting point. For the average vector method either in a 3 by 3 or a 5 by 5 environment of a point the principle direction and size is fitted with a least square algorithm. This is done for every point, except the edge points. and noise on the accuracy of determination $\mathrm{CV}_{1}$ and $\mathrm{CV}_{\mathrm{t}}$ using both methods. We discuss the pro and cons of the two methods.

\section{METHODS}

Conduction velocities determined by using the single vector and average vector method were used to analyze:

(1) An activation sequence derived from electrograms recorded with a 13 by 16 multi-electrode-grid from an in situ canine heart (Coronel et al., 2010). Stimulation was at BCL $600 \mathrm{~ms}$ from the center pair of electrodes.

(2) Simulated activation maps derived from activation times for grids of varying size and varying anisotropic ratios following stimulation from a central site. Activation times at the recording sites of the different grids were computed from elliptical isochronal lines with diagonal long and short axis. Grid-sizes from 8 by 8 to 32 by 32 with anisotropic ratios of $1.2,1.5,2$, and 3 were investigated.

For the "single vector" method a Matlab program was used. It selects a point at approximately $80 \%$ of the distance between the stimulation site to the border for the longitudinal direction, because closer to the edge one cannot see if the spread is still homogeneous. The activation in the transversal direction may be influenced by activation via deeper layers, therefore transversal velocity is often estimated from a shorter vector and 70\% was used for the transversal velocity. The stimulus point was used as the origin of the vectors (see Figure 1A).

For the average vector method local vectors were computed in a 3 by 3 and a 5 by 5 subgrid surrounding each recording site, excepting one or two rows/columns from the margins of the grid, depending on the subgrid size (see Figure 1B). The computed conduction velocity was plotted as function of the vector angle. The effect of bin-size of the vector angle was investigated by using bin sizes of 15 and $30^{\circ}$. Both seemed a-priori reasonable values as compromise between good angular resolution and enough vectors in the bins. For each combination $1 / 8,1 / 4,1 / 2$,

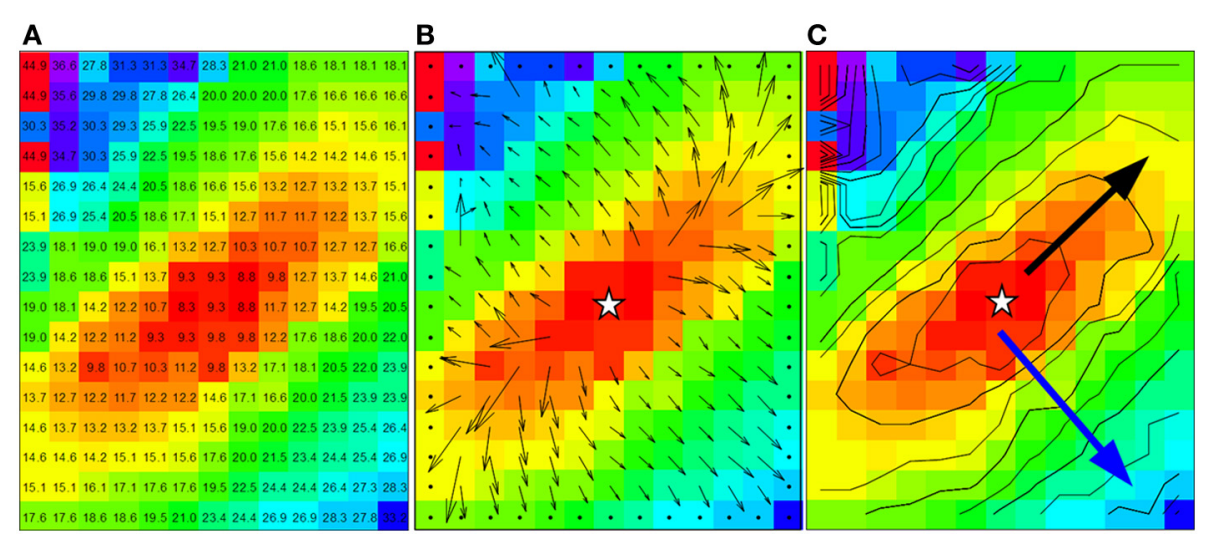

FIGURE 2 | Data from dog experiment. (A) Are the measured activation times. (B) The vector field derived from the activation times in panel a by using a 3 by 3 neighborhood least square fit. (C) Shows the approximately ellipsoid isochrones at $2 \mathrm{~ms}$ distance. The black arrow is used for the manually selected longitudinal conduction velocity $(2.8 \mathrm{~mm}$ in $6 \mathrm{~ms}$, $\mathrm{VI}=0.47 \mathrm{~m} / \mathrm{s})$ and the blue arrow for the transversal conduction velocity $(3.2 \mathrm{~mm}$ in $17 \mathrm{~ms}, V t=0.19 \mathrm{~m} / \mathrm{s})$. Star shape in $\mathbf{( B , C )}$ indicate the actual stimulus site. 
and $1 \mathrm{~ms}$ Gaussian noise was added and 1024 simulations were performed in order to simulate measurement errors. Data were rounded to $0.5 \mathrm{~ms}$ to simulate a sampling rate of $2 \mathrm{kHz}$. In total 1,638,400 (1024 simulations for 25 grid-sizes, 4 anisotropy levels, 4 noise-levels, 2 bin sizes, and 2 subgrid sizes) simulated activation maps were automatically generated and analyzed with the use of MatLab (Mathworks, Natick, MA, Potse et al., 2002).

\section{RESULTS}

\section{AVERAGE VECTOR METHOD APPLIED TO MEASURED DATA}

For the average method to give reliable results the vectors need to be collected in bins that each contain enough vectors that have very similar directions. In Figures 3A-D the vector angles of the data from Figure 2 are binned (rounded) to 15 and $30^{\circ}$, respectively. The upper panels ( $\mathrm{a}$ and $\mathrm{b}$ ) show the average conduction velocity per bin, with a gap if there was no vector in a direction that corresponds to that bin. The numbers and colors in the lower panels represent the vector angle relative to the zero angle ("east," as indicated by the cross between b and d). The red circles show the sites where the largest and smallest conduction velocities were recorded. With binning set at $15^{\circ}$ (Figure 3A), the calculated maximum conduction velocity (about $0.55 \mathrm{~m} / \mathrm{s}$ ) is recorded at a site close to the pacing site, whereas the slowest conduction velocity (about $0.11 \mathrm{~m} / \mathrm{s}$ ) is recorded at a site near the margin of the grid. Figure $3 \mathbf{B}$ shows that using bins of $30^{\circ}$, the profile of conduction velocities is more smooth than in (Figure 3A) and now leads to a maximum CV of $0.48 \mathrm{~m} / \mathrm{s}$ at $230^{\circ}$ and a minimum $\mathrm{CV}$ of $0.2 \mathrm{~m} / \mathrm{s}$ at $320^{\circ}$. If the single vector method is used the $C_{1}$ and $C V_{t}$ are 0.47 and 0.19 , respectively (see Figure 2C).

For this recorded map the number of observations in each bin varied from 0 to 27 when average vector directions were rounded to the nearest multiple of $15^{\circ}$ (Figure 4A). There is one empty bin at an angle of $225^{\circ}$. Figure $4 \mathrm{~B}$ shows that with a larger binsize there are no empty bins and the number of vectors in every bin is at least 2 .

\section{SIMULATIONS}

With the set of simulated map the effect of the various parameters on the accuracy of the calculated $\mathrm{CV}_{1}$ and $\mathrm{CV}_{\mathrm{t}}$ was analyzed at two preselected bin-sizes of 15 and $30^{\circ}$. Figure 5 shows a couple of representative simulated activation vector maps.

The simulation results for one set of values are shown in Figure 6. An anisotropic ratio of 2 and a noise level of $0.5 \mathrm{~ms}$ was used; Input $C_{1}$ and $C V_{t}$ were set at 0.9 and $0.45 \mathrm{~m} / \mathrm{s}$, respectively, and activation times and maps were generated for each grid size ranging from 8 by 8 to 32 by $32 . \mathrm{CV}_{1}$ and $\mathrm{CV}_{\mathrm{t}}$ were then calculated with the two methods. Compared to the single vector method, the
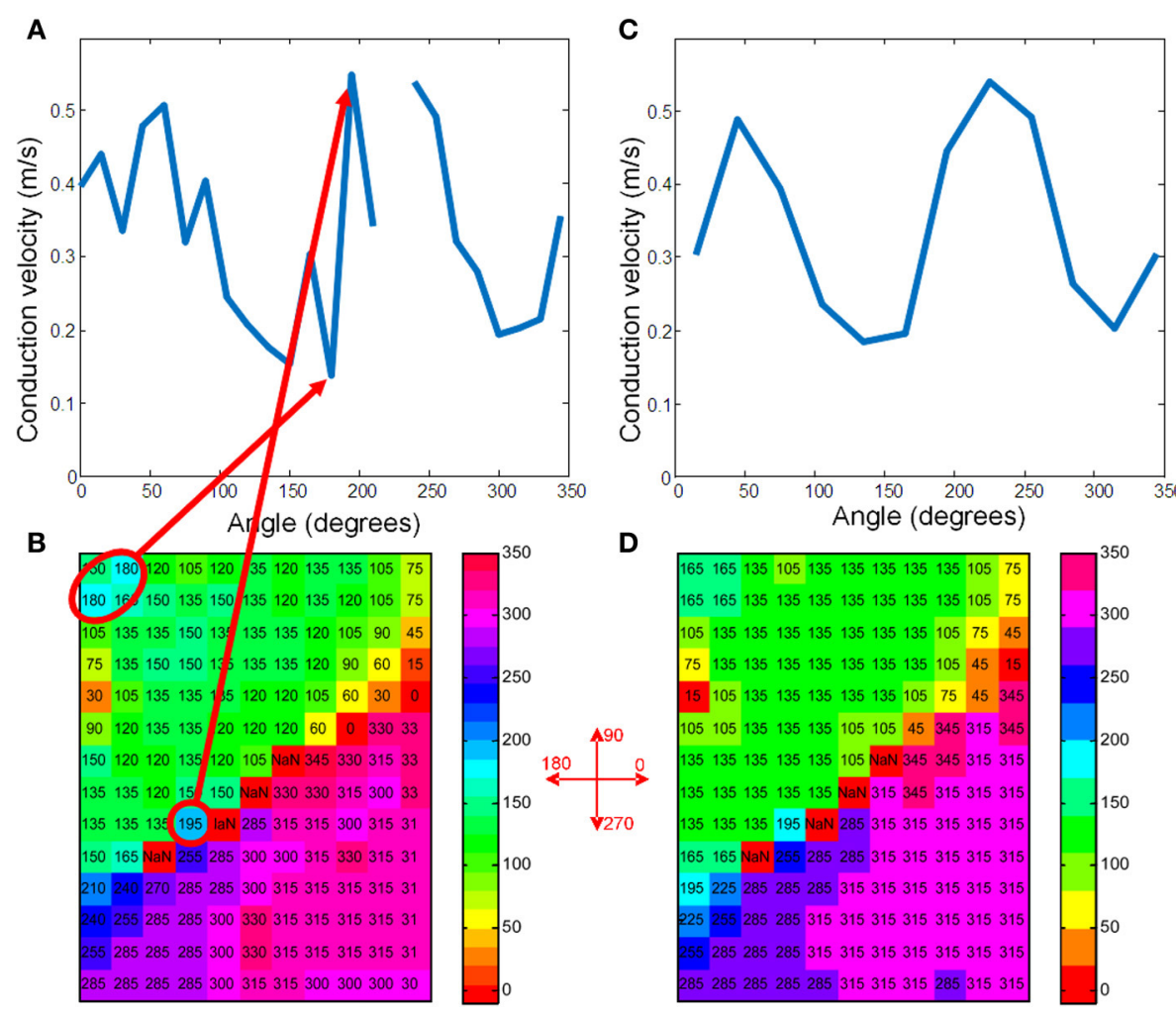

FIGURE 3 | (A) Shows the average conduction velocities in the vector-bins for the map of Figure 2 when using $15^{\circ}$ bins. (C) Shows the raw data with the vectors rounded to $15^{\circ}$. The grid positions that correspond to the highest and lowest velocities are marked with a red ellipse. Definition of the angles is indicated in between panels (B) and (D). (B,D) Give the same information for $30^{\circ}$ bins. The two maxima of the sinusoidal curve correspond to the longitudinal velocity and the minima to the transversal velocity (Bayly et al., 1998). 

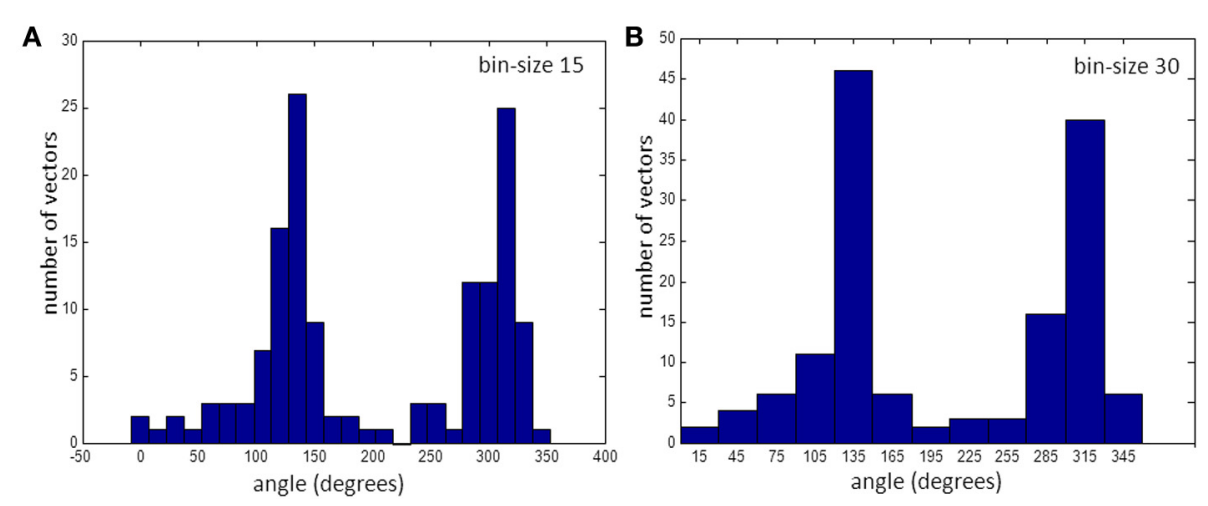

FIGURE 4 | Histogram of vectors in bins. (A) for $15^{\circ}$ bins and (B) for $30^{\circ}$. Note that the bin at $225^{\circ}$ is empty. (See also gap in Figure $3 \mathbf{A}$ ).

\section{A $16 \times 16 / 1.2 / 0.125$}

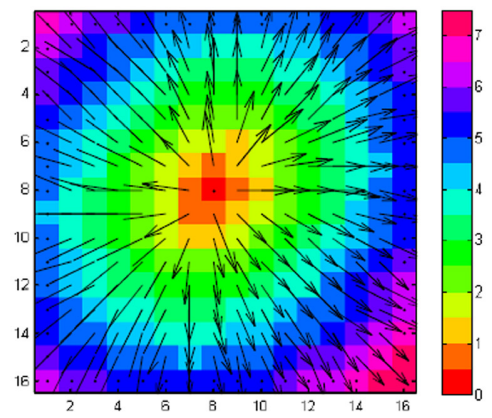

C $8 \times 8 / 2.0 / 0.5$

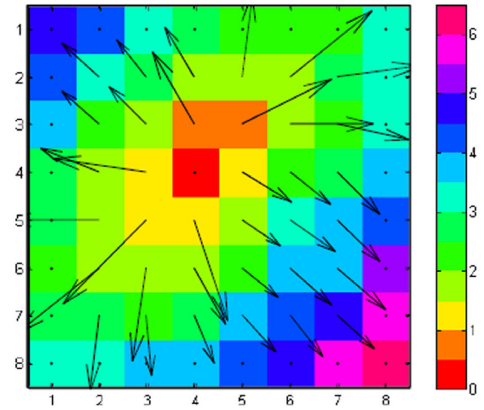

FIGURE 5 | Examples of grid sizes and anisotropic conduction velocity ratios. Numbers indicate grid size/anisotropy ratio/added noiselevel in ms. In panel (A) an example of a 16 by 16 grid is shown for an anisotropy ratio of $1.210 .9 \mathrm{~m} / \mathrm{s}$ longitudinal and $0.75 \mathrm{~m} / \mathrm{s}$ transversal) with only $0.125 \mathrm{~ms}$ gausian noise added to the simulated

\section{B $16 \times 16 / 2.0 / 0.125$}

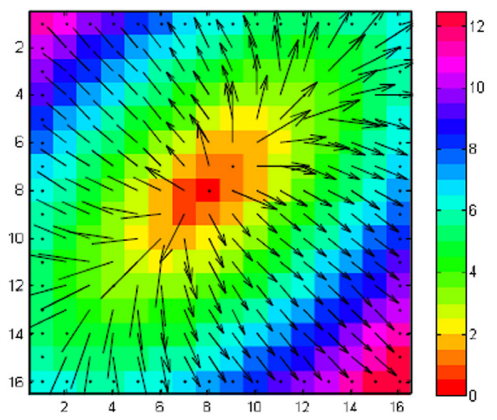

D $32 \times 32 / 2.0 / 0.5$

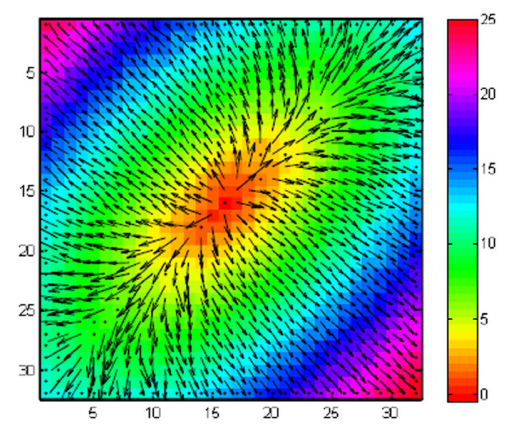

activation times. Panel (B) shows another example, this time for a anisotropy ratio of $2(0.9 \mathrm{~m} / \mathrm{s}$ longitudinal, $0.45 \mathrm{~m} / \mathrm{s}$ transversal). Panel (C) shows a simulation for a smaller grid ( 8 by 8 ) and panel (D) for a larger grid (32 by 32). In (C) and (D) also the simulated noise is larger with a standard deviation of $0.5 \mathrm{~ms}$. "average vector" (blue and green lines) method has a larger overestimation of the longitudinal velocity for almost all grid sizes at a bin size of $15^{\circ}$, except for very large grids (approximately 22 by 22 ) and using 5 by 5 subgrids (Figure 5A). For grid-sizes smaller than about 16 by 16 for a bin-size of $30^{\circ}$ (Figure 6B) values are also overestimated. The value used for the simulation is not within 1 SD from the mean for the average method for a bin size of 15 , except for the smallest grid sizes where the SD is largest. The estimated values converge to the input value at grid sizes larger than 20, but at the same time the SD also becomes smaller and the true value does not come in range. The single vector method is closer to the actual value but has a larger SD than the 5 by 5 subgrid for larger grid sizes. Calculated $\mathrm{CV}_{t}$ is closer to the actual value than the $\mathrm{CV}_{1}$ for both methods but the SD for the average method is so small that there is consistent overestimation. 

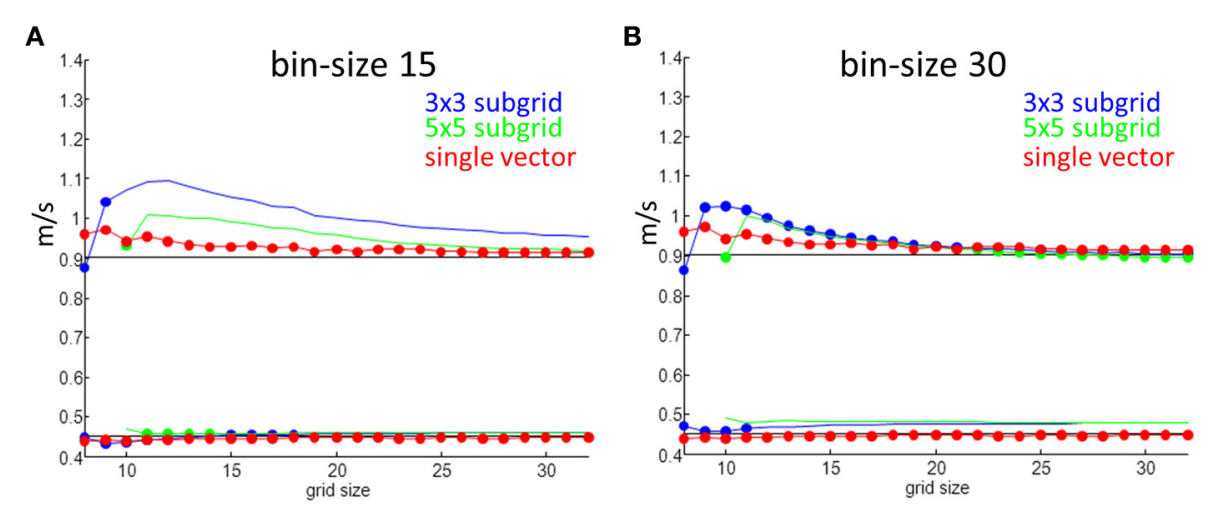

FIGURE 6 | Velocity measurements as a function of grid size. Red are estimates from the single vector method, blue average method for a 3 by 3 subgrid and green for a 5 by 5 subgrid. Dots indicate that the true value is within one SD of the mean value. (A) Shows results for a bin size of $15^{\circ}$ and (B) is for a bin size of $30^{\circ}$

Overestimation in the longitudinal direction comes from measurement errors that result in one or two accidentally large vectors that are put in an (almost) empty bin. Overestimation in the transverse direction results from a large number of vectors that are a mix of transversal and longitudinal and therefore longer being put in the same bin as the pure transversal ones.

\section{MINIMUM SIZE TO PREVENT EMPTY BINS}

At a bin-size of $15^{\circ}$ and small grid-sizes it may happen that no vector is present in some of the bins (see Figure 4A), including those bins containing the angle representing fiber direction or, less likely, orthogonal to that. At large anisotropic ratios the chance of an empty bin in the direction of the fiber is larger than with a small anisotropic ratio. Thus, estimation of $\mathrm{CV}_{1}$ with the average vector method is more difficult and less reliable with smaller bin sizes, small grid sizes and larger anisotropy.

By varying the anisotropic ratio from 1.2 to 3 it was possible to estimate the minimum required size of a square grid that has a chance of less than $5 \%$ that the bin in the fiber direction contains no vector at all (1024 tests). The table shows the results of these simulations. The table shows for instance that a grid-size of at least 14 by 14 at an anisotropy-ratio of 2 and a noise-level of $0.5 \mathrm{~ms}$ is required to keep the chance below $5 \%$ that no vector is present in the bin representing the fiber direction (see Table 1). Required grid size increases with anisotropy. The addition of a realistic level of noise in the measurements again increases the minimum required size of the grid in order to prevent empty bins in the angle of the fiber direction.

The single vector method is less sensitive to grid size, noise, and the degree of anisotropy. In all cases a mean closer than one $\mathrm{SD}$ from the actual value of $\mathrm{CV}_{\mathrm{l}}$ and $\mathrm{CV}_{\mathrm{t}}$ was computed.

\section{DISCUSSION}

\section{POSSIBLE PROBLEMS WITH THE AVERAGE VECTOR METHOD APPLIED TO REAL DATA}

If the "average vector" method is applied to real data, a number of problems may arise. The most important one in the example given is that neither the direction of the longitudinal (fastest) conduction nor the transversal conduction (slowest) conduction are along the long and short axis of the elliptical pattern. From Figure 3C it can be appreciated that the "longitudinal conduction velocity" (at $195^{\circ}$ ) is based on a single $3 \times 3$ subgrid observation that is very close to the site of stimulation. Close to the site of stimulation "latency" occurs which contorts activation pattern. Koller et al. (1995) on the other hand, the "transversal conduction velocity" is an average of two $3 \times 3$ subgrids in an area (see Figure 2) where isochronal lines are not equidistant. This area would have been excluded from analysis during single vector analysis. Another problem is that some bins are empty (Figure 3A). Unfortunately the bin most likely to be empty is the one in the direction of the longitudinal CV. Even if it is not empty, the number of local activation vectors is in general low, resulting is a large standard deviation. Contamination with vectors not purely in the direction of the fibers will result in a underestimation of the actual conduction velocity. In contrast, the transversal directions at 135 and $315^{\circ}$ do contain a large number of vectors, but many of these are at sites at which activation is the result of a composite of transverse and longitudinal conduction. This results in a measurement with a small SD, but it may also suffer from an overestimation.

\section{ERRORS BY NON-ELLIPTICAL SPREAD OF ACTIVATIONS}

The methods were compared during central stimulation under conditions in which it was assumed that no independent information on fiber direction is available. The single vector method is not applicable during other activation patterns, because the information on fiber direction cannot be derived from the activation map. Vector methods were originally developed to quantify the degree of heterogeneity in activation timing during anisotropic conduction (Lammers et al., 1990). Under those circumstances the average vector method may allow estimation of $\mathrm{CV}_{1}$ and $\mathrm{CV}_{\mathrm{t}}$. One should, however, be aware that breakthrough patterns may yield unrealistic high $\mathrm{CV}_{1}$ values, because transmural rotation of myocardial fibers affects the epicardial activation pattern (Frazier et al., 1988; Schalij et al., 1992). Therefore, parts of the activation map where activation in deeper layers affects the layer where recordings are made, should not be used for analysis. In this 
Table 1 | Minimum required size of grid to have a $95 \%$ chance of having at least one vector in the bin representing the direction of the fibers.

\begin{tabular}{|c|c|c|c|c|c|c|c|c|c|c|c|c|c|c|c|c|}
\hline \multirow[b]{3}{*}{ Anisotropy } & \multicolumn{8}{|c|}{3 by 3} & \multicolumn{8}{|c|}{5 by 5} \\
\hline & \multicolumn{4}{|c|}{$15^{\circ}$ bins } & \multicolumn{4}{|c|}{$30^{\circ}$ bins } & \multicolumn{4}{|c|}{$15^{\circ}$ bins } & \multicolumn{4}{|c|}{$30^{\circ}$ bins } \\
\hline & 1,2 & 1,5 & 2 & 3 & 1,2 & 1,5 & 2 & 3 & 1,2 & 1,5 & 2 & 3 & 1,2 & 1,5 & 2 & 3 \\
\hline Noise $1 / 8 \mathrm{~ms}$ & 10 & 13 & 11 & 13 & 9 & 9 & 11 & 19 & 11 & 14 & 20 & 31 & 11 & 11 & 13 & 19 \\
\hline Noise $1 / 4 \mathrm{~ms}$ & 10 & 13 & 13 & 15 & 9 & 9 & 12 & 19 & 12 & 14 & 20 & 25 & 11 & 11 & 13 & 19 \\
\hline Noise $1 / 2 \mathrm{~ms}$ & 11 & 12 & 14 & 17 & 11 & 11 & 13 & 19 & 12 & 14 & 18 & 23 & 11 & 11 & 13 & 19 \\
\hline Noise $1 \mathrm{~ms}$ & 12 & 13 & 15 & 19 & 11 & 11 & 13 & 17 & 12 & 14 & 17 & 23 & 11 & 11 & 13 & 17 \\
\hline
\end{tabular}

Numbers are for square grids, so "10" means at least a 10 by 10 grid.

simulation study a single layer with one global fiber orientation was assumed. For an automatic method based on the average vector method deviations from elliptical spread should also be detected automatically.

\section{OVERESTIMATES AND UNDERESTIMATES OF VELOCITIES USING THE AVERAGE VECTOR METHOD}

Both methods perform relatively well for the determination of transverse conduction velocity, and are not dependent on grid size, bin size (average vector method) and anisotropy. For small anisotropy and small grid sizes (<about $20 \times 20$ ) the average vector method underestimates transverse $\mathrm{CV}_{\mathrm{t}}$ with more than 1 SD. This effect is larger for a bin size of 15 than for $30^{\circ}$. Longitudinal $\mathrm{CV}$ is often overestimated at a bin size of 15 by the average vector method when the grid size is low $(<$ about $20 \times 20$ ). When the anisotropic ratio is large, the overestimation at smaller sizes is larger and also the chance that no vectors are in the fastest bin. Using a 5 by 5 grid in general improves the quality of the estimate, but larger grid sizes are required to be able to reliably measure at all. Using $30^{\circ}$ bins instead of $15^{\circ}$ gives better overall results.

\section{LIMITATIONS OF THIS STUDY}

A limitation of this study is that these simulations assume that the activation spreads from a single stimulus point in the middle of the grid.

The assumption in this paper is that the myocardium is homogeneously anisotropic. As the example in Figure 2 shows, this is often not the case in real life. This is a minor problem for the "single vector" method, but for the "average vector" method it will result in a large number of vectors placed in the wrong bin. Worse, artifacts that result in overly long or small vectors in inhomogeneous parts can dominate the real vectors when the average is calculated from a small number of vectors. This will typically happen with the vectors in the direction of the fibers when the anisotropic ratio is 1.5 or more. The results obtained will therefore definitely be too large in case of the "average vector" method.

For the simulation experiments the direction of the longitudinal direction was known (i.e., $45^{\circ}$ ). Bin boundaries were selected in such a way that this $45^{\circ}$ was in the middle of the bin. So, for $30^{\circ}$ bins boundaries were $0,30,60, \ldots$ and for the $15^{\circ}$ bins -7.5 , $7.5,22.5,37.5,62.5, \ldots$ For experiments in real hearts, this information is in general not known; using this knowledge may have positively skewed the results for the average method.

\section{CLINICAL APPLICATIONS OF MULTIELECTRODE MAPPING}

Multielectrode mapping with regular grid electrodes in patients has been reported to determine the arrhythmogenic site of a tachycardia (Elvan et al., 2011) during open heart surgery or to assess the integrity of lines of conduction during minimal invasive procedures for AF (de Groot et al., 2012). Catheter approaches with 2D (Elvan et al., 2009) or 3D (Potse et al., 2004) multi electrode catheters have been reported as well, but in both these cases the inter-electrode distances are not constant, making the use of the average method difficult, if not impossible.

\section{CONCLUSIONS}

This study compared two methods for the determination of conduction velocity.

The average vector method is a fast and easy tool to estimate the conduction velocities in myocardium. This method is often used in optical mapping experiments (Mironov et al., 2008) in which relatively large grid sizes are used $(100 \times 100)$. We demonstrate that under these conditions the method is highly reliable (after stimulation in the center of the grid), although a systematic underestimation of $\mathrm{CV}_{1}$ can be expected. When using small binsizes the average vector method will overestimate the longitudinal velocities. For larger grid-sizes it can overestimate the transversal velocities. Using a 5 by 5 subgrid requires larger grid sizes than 3 by 3 , but the standard deviation of the results is smaller, and for smaller bin size the results are closer to the true value. Using larger subgrids does not fully solve the overestimation.

The single vector method performs better than the average vector method when grid size is small, bin size is small, sampling rate is low and anisotropy is large. This is at least in part related to the fact that (1) the identification of fiber direction allows selection of a single vector in the correct direction (and perpendicular to it); (2) the intrinsic assumption that $\mathrm{CV}_{\mathrm{t}}$ is measured at right angles from $\mathrm{CV}_{1}$; and (3) that the largest possible distance over which linear conduction takes place is selected. The latter obviates the influence of breakthrough activation and allows greater time resolution (less influence of sampling rate). The single vector method is the preferred method when manual processing is an option.

\section{REFERENCES}

Arita, M., and Kiyosue, T. (1983). Modification of "depressed fast channel dependent slow conduction" by lidocaine and verapamil in the presence or absence of catecholamines. Evidence for alteration of preferential ionic channels for slow conduction. Jap. Circ. J. 47, 68-81. 
Bayly, P., KenKnight, B., Rogers, J., Hillsley, R., and Ideker, R. (1998). Estimation of conduction velocity vector fields from epicardial mapping data. IEEE Trans. Biomed. Eng. 45, 563-571.

Coronel, R., Lau, D., Sosunov, E., Janse, M., Danilo, P., Anyukhovsky, E., et al. (2010). Cardiac expression of skeletal muscle sodium channels increases longitudinal conduction velocity in the canine 1-week myocardial infarction. Heart Rhythm 7, 1104-1110. doi: 10.1016/j.hrthm.2010.04.009

Coronel, R., Wilms-Schopman, F., Opthof, T., and Janse, M. (2009). Dispersion of repolarization and arrhythmogenesis. Heart Rhythm 6, 537-543. doi: 10.1016/j.hrthm.2009.01.013

de Bakker, J., van Capelle, F., Janse, M., Tasseron, S., Vermeulen, J., de Jonge, N., et al. (1993). Slow conduction in the infarcted human heart. 'Zigzag' course of activation. Circulation 88, 915-926. doi: 10.1161/01.CIR.88.3.915

de Groot, J., Driessen, A., van Boven, W., Krul, S., Linnenbank, A., Jackman, W., et al. (2012). Epicardial confirmation of conduction block during thoracoscopic surgery for atrial fibrillation-a hybrid surgical-electrophysiological approach. Minim. Invasive Ther. Allied Technol. 21, 293-301. doi: 10.3109/13645706.2011. 615329

Elvan, A., Linnenbank, A., van Bemmel, M., Misier, A. R., Delnoy, P., Beukema, W., et al. (2009). Dominant frequency of atrial fibrillation correlates poorly with atrial fibrillation cycle length. Circ. Arrhythm. Electrophysiol. 2, 634-644. doi: 10.1161/CIRCEP.108.843284

Elvan, A., Sie, H., Misier, A. R., Linnenbank, A., Delnoy, P., and de Bakker, J. (2011). Mapping and surgical ablation of focal epicardial left ventricular tachycardia. Case Rep. Cardiol. 2011:471397. doi: 10.1155/2011/471397

Frazier, D., Krassowska, W., Chen, P., Wolf, P., Danieley, N., Smith, W., et al. (1988). Transmural activations and stimulus potentials in three-dimensional anisotropic canine myocardium. Circ. Res. 63, 135-146. doi: 10.1161/01.RES. 63.1.135

Hoogendijk, M., Potse, M., Linnenbank, A., Verkerk, A., den Ruijter, H., van Amersfoorth, S., et al. (2010). Mechanism of right precordial ST-segment elevation in structural heart disease: excitation failure by current-to-load mismatch. Heart Rhythm 7, 238-248. doi: 10.1016/j.hrthm.2009.10.007

Janse, M., and Wit, A. (1989). Electrophysiological mechanisms of ventricular arrhythmias resulting from myocardial ischemia and infarction. Physiol. Rev. 69, 1049-1169.

Kawara, T., Derksen, R., de Groot, J., Coronel, R., Tasseron, S., Linnenbank, A., et al. (2001). Activation delay after premature stimulation in chronically diseased human myocardium relates to the architecture of interstitial fibrosis. Ciculation 104, 3069-3075. doi: 10.1161/hc5001.100833

Kleber, A., Janse, M., Wilms-Schopman, F., Wilde, A., and Coronel, R. (1986). Changes in conduction velocity during acute ischemia in ventricular myocardial isolated porcine heart. Circulation 73, 189-198. doi: 10.1161/01.CIR.73.1.189

Koller, B., Karasik, P., Solomon, A., and Franz, M. (1995). Prolongation of conduction time during premature stimulation in the human atrium is primarily caused by local stimulus response latency. Eur. Heart J. 16, 1920-1924.

Lammers, W., Schalij, M., Kirchhof, C., and Alessie, M. (1990). Quantification of spatial inhomogeneity in conduction and initiation of reentrant atrial arrhythmias. Am. J. Physiol. 259, H1254-H1263.

Mines, G. (1914). On circulating excitations in heart muscles and their possible relation to tachycardia and fibrillation. Trans. R. Soc. Can. 4, 43-53.

Mironov, S., Jalife, J., and Tolkacheva, E. (2008). Role of conduction velocity restitution and short-term memory in the development of action potential duration alternans in isolated rabbit hearts. Circulation 118, 17-25. doi: 10.1161/ CIRCULATIONAHA.107.737254
Peters, N., Coromilas, J., Severs, N., and Wit, A. (1997). Disturbed connexin43 gap junction distribution correlates with the location of reentrant circuits in the epicardial border zone of healing canine infarcts that cause ventricular tachycardia. Circulation 95, 988-996. doi: 10.1161/01.CIR.95.4.988

Potse, M., Linnenbank, A., and Grimbergen, C. (2002). Software design for analysis of multichannel intracardial and body surface electrocardiograms. Comput. Methods Programs Biomed. 69, 225-236. doi: 10.1016/S0169-2607(02) 00014-7

Potse, M., van Dessel, P., Linnenbank, A., Grimbergen, C., van Hemel, N., and de Bakker, J. (2004). Properties of unipolar electrograms recorded with a multielectrode basket catheter. J. Electrocardiol. 37, 1-10. doi: 10.1016/j.jelectrocard. 2003.10.008

Remme, C., Verkerk, A., Nuyens, D., van Ginneken, A., van Brunschot, S., Belterman, C., et al. (2006). Overlap syndrome of cardiac sodium channel disease in mice carrying the equivalent mutation of human SCN5A1795insD. Circulation 114, 2584-2594. doi: 10.1161/CIRCULATIONAHA.106. 653949

Schalij, M., Lammers, W., Rensma, P., and Alessie, M. (1992). Anisotropic conduction and reentry in perfused epicardium of rabbit left ventricle. Am. J. Physiol. 263, 1466-1478.

Spach, M., Miller, W., Geselowitz, D., Barr, R., Kootsey, J., and Johnson, E. (1981). The discontinuous nature of propagation in normal canine cardiac muscle. Evidence for recurrent discontinuities of intracellular resistance that affect the membrane currents. Circ. Res. 48, 39-54. doi: 10.1161/01.RES.48.1.39

Valdivia, C., Chu, W., Pu, J., Foell, J., Haworth, R., Wolff, M., et al. (2005). Increased late sodium current in myocytes from a canine heart failure model and from failing human heart. J. Mol. Cell. Cardiol. 38, 475-483. doi: 10.1016/j.yjmcc. 2004.12.012

Weber, K., and Brilla, C. (1991). Pathological hypertrophy and cardiac interstitium. Fibrosis and renin-angiotensin-aldosterone system. Circulation 83, 1849-1865. doi: 10.1161/01.CIR.83.6.1849

Wiegerinck, R., Verkerk, A., Belterman, C., van Veen, T., Baartscheer, A., Opthof, T., et al. (2006). Larger cell size in rabbits with heart failure increases myocardial conduction velocity and QRS duration. Circulation 48, 806-813. doi: 10.1161/CIRCULATIONAHA.105.565804

Wilders, R., Wagner, M., Golod, D., Kumar, R., Wang, Y., Goolsby, W., et al. (2000). Effects of anisotropy on the development of cardiac arrhythmias associated with focal activity. Pfluegers Arch. 441, 301-312. doi: 10.1007/s004240000413

Conflict of Interest Statement: The authors declare that the research was conducted in the absence of any commercial or financial relationships that could be construed as a potential conflict of interest.

Received: 25 March 2014; accepted: 25 June 2014; published online: 22 July 2014. Citation: Linnenbank AC, de Bakker JMT and Coronel R (2014) How to measure propagation velocity in cardiac tissue: a simulation study. Front. Physiol. 5:267. doi: 10.3389/fphys.2014.00267

This article was submitted to Cardiac Electrophysiology, a section of the journal Frontiers in Physiology.

Copyright (C) 2014 Linnenbank, de Bakker and Coronel. This is an open-access article distributed under the terms of the Creative Commons Attribution License (CC BY). The use, distribution or reproduction in other forums is permitted, provided the original author(s) or licensor are credited and that the original publication in this journal is cited, in accordance with accepted academic practice. No use, distribution or reproduction is permitted which does not comply with these terms. 